\title{
Avaliação do uso de medicamentos por funcionários terceirizados de um centro de ensino em saúde
}

\section{Evaluation of the use of medicines by outsourced employees of a health education center}

\author{
Shirley Mareza de Souza Santos \\ Universidade Federal do Recôncavo da Bahia - UFRB - Santo Antonio de Jesus - BA - Brasil \\ marezass@hotmail.com \\ Djanilson Barbosa dos Santos \\ Universidade Federal do Recôncavo da Bahia - UFRB - Santo Antonio de Jesus - BA - Brasil \\ djanilsonb@gmail.com \\ Luiz Antonio Favero Filho \\ Universidade Federal do Espírito Santo - UFES - São Mateus - ES - Brasil \\ luizfavero@gmail.com
}

\section{Resumo}

A Organização Mundial da Saúde (OMS) refere a automedicação como um dos graves problemas de saúde pública. No Brasil a automedicação tem sido um fator de grande preocupação, pois pode desencadear efeitos indesejáveis e levar o indivíduo a morte. Este trabalho tem por objetivo realizar uma análise do perfil de utilização de medicamentos por funcionários terceirizados de um Centro de Ciências da Saúde de uma Universidade Federal, correlacionando o perfil do usuário com a forma de utilização do(s) medicamento(s). Tratou-se de uma pesquisa quantitativa, que usou como instrumento para coleta de dados um questionário estruturado contendo 22 questões, respondidas após anuência e assinatura do termo de consentimento livre e esclarecido (TCLE). Identificou-se a prevalência de homens $(67,2 \%)$ com faixa etária de mais de 44 anos $(30,8 \%)$. Em relação à frequência da automedicação, $89,7 \%$ dos entrevistados relatam já terem realizado essa prática. Dentre os motivos que influenciaram na automedicação os mais citados foram a compra na farmácia por conta própria $(63,8 \%)$ e compra na farmácia por indicação do vendedor (32,8\%). Também foi observado que pessoas que possuem planos de saúde $(66,7 \%)$ fazem automedicação de forma mais significativa do que aquelas que não possuem $(26,3 \%)$. Os dados coletados são de grande valia na definição de estratégias voltadas à promoção do uso racional de medicamentos.
\end{abstract}

Palavras-chave: uso de medicamentos, automedicação, farmacoepidemiologia.

\section{Abstract}

The World Health Organization (WHO) refers to self-medication as a serious public health problem. Self-medication in Brazil has been a factor of great concern because it can result in undesirable effects and it can cause death. The objective of this study was to analyze the use of medications for outsourced employees of a Center of Health Sciences of a 
Federal University. It compared the user profile in the different form of consumption of the medication. This was a quantitative study, which used was a tool for data collection, a structured questionnaire containing 22 questions. The questionnaire was answered after an approved and signed the Informed Consent Form (ICF). It identifies the prevalence of men $(67,2 \%)$ who are over 44 years old $(30,8 \%)$. Regarding the frequency of selfmedication, $89.7 \%$ of respondents reported having already performed this practice. Among the factors influencing self-medication were buying at the pharmacy on their own $(63,8 \%)$, those who bought at the drugstore seeking details of the seller were $32,8 \%$. It was also noted that people who have health insurance $(66,7 \%)$ were self-medicated more significantly than those without them (26,3\%). The collected data are of great value in defining strategies for promoting the rational use of medicines.

Keywords: use of medicines, self-medication, pharmacoepidemiology.

\section{Introdução}

Os fármacos ocupam um importante papel nos sistemas sanitários, pois salvam vidas e melhoram a saúde (MARIN, 2003). As diretrizes farmacoterápicas ajustadas para a condição clínica do indivíduo são elementos essenciais para determinar o emprego de medicação. Contudo, é importante destacar que a prescrição e o uso de medicamentos são influenciados por fatores de natureza cultural, social, econômica e política (FAUS, 2000; PERINI et al., 1999).

Millian et al. (2003) definiram automedicação como o consumo de um fármaco (que podem ser medicamentos industrializados ou caseiros) sem orientação ou prescrição de profissionais habilitados, onde o próprio indivíduo decide o produto que será utilizado. Inclui-se nessa denominação geral de automedicação também a prescrição (ou orientação) de medicamentos por pessoas leigas no assunto, como amigos, familiares ou balconistas da farmácia. Outro termo utilizado é a automedicação orientada, que se refere à reutilização de receitas antigas sem que elas tenham sido emitidas para uso contínuo. Desta forma, a automedicação é compreendida como um procedimento caracterizado pela iniciativa de um doente ou seu responsável em utilizar um produto que trará benefícios no tratamento de doenças ou no alívio de sintomas. (PAULO; ZANINE, 1988).

O uso de medicamentos e a automedicação estão sendo amplamente estudados tanto no Brasil quanto em outros países. Uma revisão de literatura observou que a maior parte dos estudos sobre práticas de automedicação foram realizados no Brasil, seguidos por Índia, Paquistão e Nigéria. Esta revisão apontou que 31\% dos estudos investigaram a automedicação com antimicrobianos, enquanto 54\% não abordaram uma área específica (LIMAYE, 2017). No Brasil a automedicação é um fato que vem sendo de grande preocupação e estimula debates na comunidade científica devido a sua frequência na população brasileira (BOCK; TARANTINO, 2001). Estudos como os de Barros (1997) e Nascimento (2003) demonstram que aproximadamente $35 \%$ dos medicamentos consumidos no Brasil são feitos por meio da automedicação.

A revisão sistemática realizada por Domingues et al (2015), demonstrou que em países em desenvolvimento a prevalência da automedicação é maior que os resultados dos estudos praticados na Espanha. Nos países desenvolvidos a automedicação vem crescendo constantemente, devido a fácil aquisição de medicamentos com venda indiscriminada nas farmácias. Nesses países, entretanto, existem rígidos controles estabelecidos pelas agências reguladoras, somado ao crescente envolvimento dos farmacêuticos com a orientação dos usuários de medicamentos, tornando menos problemática a prática da automedicação (ARRAIS, 1997).

O setor privado é o principal responsável pelo fornecimento de medicamentos a população e a comercialização de medicamentos nas farmácias em geral está nas mãos 
de leigos, proprietários e balconistas (NAVES et al, 2010). Estudos realizados em farmácias observaram alta prevalência de indicação de fármacos por balconistas (CAMPOS et al. 1985; SOIBELMAN et al. 1986). De acordo com o Sistema Nacional de Informações Tóxico-Farmacológicas (Sinitox), os medicamentos ocupam o primeiro lugar entre os agentes causadores de intoxicações em seres humanos. Somente em 2016, segundo este sistema, os medicamentos provocaram $34,04 \%$ do total de intoxicações registradas no país (FIOCRUZ, 2016).

O consumo adequado de fármacos pode ser afetado positivamente pelas políticas nacionais promovendo a regulamentação do suprimento e a disponibilização racional de medicamentos essenciais, implicando 0 acesso ao diagnóstico e prescrição por profissionais habilitados. Entretanto, o consumo pode ser influenciado negativamente pelo acesso sem barreiras e pela promoção e publicidade de medicamentos, que muitas vezes estimulam a utilização desnecessária e irracional (NAVES et al., 2010).

É de suma importância ressaltar que a automedicação irracional é um acontecimento nocivo à saúde individual e coletiva, já que nenhum medicamento é inócuo ao organismo, podendo ter consequências, como efeitos indesejáveis, enfermidades iatrogênicas e mascaramento de doenças evolutivas, representando, deste modo, um problema a ser prevenido (ARRAIS, 1997). Os principais erros que podem desencadear reações de maior complexidade são: medicamento impróprio, dose errada, frequência inadequada, período insuficiente ou demasiado de consumo e combinação inadequada com outros fármacos provocando interação indesejável (NASCIMENTO, 2003).

Os prejuízos mais frequentes decorrentes da automedicação são gastos supérfluos, atraso no diagnóstico e na terapêutica adequada, reações adversas ou alérgicas, intoxicação, agressões ao sistema digestivo e em situações mais graves internação hospitalar a até mesmo a morte (PAULO; ZANINE, 1988; ARRAIS, 1997). De acordo com dados da OMS, os hospitais gastam de $15 \%$ a $20 \%$ de seus orçamentos para lidar com as complicações causadas pelo mau uso de medicamentos. Os modelos tradicionais de prática farmacêutica têm mostrado pouca eficácia respeitando a morbimortalidade relacionada a medicamentos (CIPOLLE et al., 2000). A prevalência e custos de morbimortalidade relacionada a medicamentos são relevantes para os gestores dos sistemas de saúde, pacientes e sociedade como um todo (JOHNSON; BOOTMAN, 1995; MALHOTRA et al., 2001). O estudo de Vilarino (1998) demonstrou uma superavaliação da eficácia dos medicamentos pela população, já que no estudo $46,5 \%$ dos entrevistados não cogitam uma terapêutica que não seja farmacológica.

Diante dessa conjuntura, a Organização Mundial de Saúde (WORLD HEALTH ORGANIZATION, 1987) refere à automedicação como um dos graves problemas de saúde pública. Portanto, é possível pensar em medidas preventivas que contribuam para a diminuição diária de riscos causados pela automedicação e consequentemente tornar aparente a conscientização da população quanto ao perigo dos efeitos adversos que certos medicamentos podem causar. Dessa forma, deve-se estar atento à possibilidade de utilizar a automedicação como um instrumento para a promoção da saúde, desde que devidamente direcionada diante de programas institucionais que visem a conferir maior grau de autonomia ao paciente frente à sua medicação, o que já vem ocorrendo experimentalmente em países de Primeiro Mundo (KELLY, 1994).

Sendo assim, o presente trabalho tem por objetivo realizar uma análise do perfil de utilização de medicamentos por funcionários terceirizados de um Centro de Ciências da Saúde de uma Universidade Federal localizada no interior do Nordeste, correlacionando o perfil do usuário com a forma de utilização do(s) medicamento(s).

\section{Metodologia}


Os 58 funcionários terceirizados do Centro de Ciências da Saúde da Universidade Federal do Recôncavo da Bahia (CCS/UFRB), em Santo Antônio de Jesus (BA), foram convidados a participar da pesquisa. Todos os participantes tomaram ciência e posteriormente, após concordância em participar, assinaram o termo de consentimento livre e esclarecido. O nível de conhecimento dos trabalhadores terceirizados sobre medicamentos foi medido por um estudo transversal realizado através de entrevistas que ocorreram conforme disponibilidade de horário dos entrevistados, nos meses de novembro de 2013 a janeiro de 2014. Essas entrevistas foram realizadas com o auxílio de um questionário composto de 22 questões relativas às características sociodemográficas da população em estudo, conhecimento sobre o uso de medicamentos e características do acesso à saúde, sendo que não existiu limite de tempo para o período recordatório. Algumas das questões permitiam ao entrevistado escolher mais de uma resposta. As entrevistas ocorreram em local reservado, com a presença apenas do entrevistado e da entrevistadora, nas dependências do CCS/UFRB.

Os dados coletados foram reunidos, codificados em banco de dados e arquivados no programa SPSS versão 20. Observaram-se as seguintes categorias de variáveis:

a)Características sócio demográficas;

b)Características sobre o uso de medicamentos;

c) Características sobre o acesso à saúde.

A execução deste projeto foi autorizada pelo Comitê de Ética da Universidade Federal do Recôncavo da Bahia. (Código do parecer 416.587).

\section{Resultados}

Foram entrevistados 58 funcionários terceirizados do CCS/UFRB, ou seja, não ocorreu nenhuma recusa em fazer parte do estudo. A distribuição dos entrevistados por gênero, faixa etária, escolaridade, estado civil, número de moradores de cada residência e renda familiar de cada funcionário está apresentada na Tabela 1.

Tabela 1: Características Socio-demográficas

\begin{tabular}{llc}
\hline Variável & $\mathbf{n}$ & $\%$ \\
\hline Sexo & 19 & 32,8 \\
Feminino & 39 & 67,2 \\
Masculino & & \\
Faixa Etária & 03 & 5,2 \\
18 a 23 anos & 18 & 31,0 \\
24 a 30 anos & 14 & 24,1 \\
31 a 37 anos & 07 & 12,1 \\
38 a 43 anos & 16 & 27,6 \\
44 ou mais anos & & \\
Escolaridade & 0 & 0 \\
Analfabeto & 10 & 17,2 \\
Entre 1 e 8 anos de estudo & 48 & 82,8 \\
9 ou mais anos de estudo & & \\
Estado Civil & 26 & 44,8 \\
Casado & 16 & 27,6 \\
Solteiro & 0 & 0 \\
Viúvo & 2 & 3,4 \\
Divorciado & 14 & 24,1 \\
União estável & & \\
Número de moradores por & & \\
residência & 2 & 3,4 \\
1 pessoa & 13 & 22,4 \\
2 pessoas & 16 & 27,6 \\
3 pessoas &
\end{tabular}




\begin{tabular}{lcc}
4 pessoas & 13 & 22,4 \\
5 ou mais pessoas & 14 & 24,1 \\
Renda mensal & & \\
1 salário mínimo & 10 & 17,2 \\
2 salários mínimos & 23 & 39,7 \\
3 salários mínimos & 14 & 24,1 \\
4 salários mínimos ou mais & 11 & 19,0 \\
\hline \multicolumn{2}{c}{ Fonte: Pesquisa de campo (2014) }
\end{tabular}

A aplicação do questionário também permitiu a obtenção de dados sobre o perfil de saúde. Dos 58 entrevistados, $89,7 \%$ responderam a questão relacionada à prática de automedicação; $51,7 \%$ dos entrevistados afirmaram ter plano de saúde, sendo que $19 \%$ passaram por uma consulta médica nos últimos 12 meses, 22,4\% por duas, 17,2\% por três e $32,8 \%$ por mais de três consultas médicas nos últimos 12 meses, enquanto 8,6\% não passaram por este atendimento. Quando questionados sobre terem ido a alguma farmácia nos últimos 4 meses, 24,1\% responderam que foram mais de 3 vezes, $8,6 \%$ foram 3 vezes, $19 \%$ duas vezes, $19 \%$ uma vez e $29,3 \%$ relatam não terem buscado alguma farmácia nos últimos 4 meses. Outra questão abordada foi o uso de plantas medicinais: $77,6 \%$ afirmam que recorrem ao uso de folhas, chás e plantas medicinais, enquanto $22,4 \%$ não utilizam desta terapêutica. Dos que utilizam desta terapêutica, $17,8 \%$ o fazem por indicação de amigo/familiar/vizinho; $6,7 \%$ pelo custo e $66,7 \%$ por acreditarem que não fazem mal à saúde. Finalmente, quando questionados sobre ações para melhoria de saúde, $72,5 \%$ afirmaram que reduziram o uso de sal para melhorar a saúde; $56,9 \%$ reduziram o uso de açúcar; $70,6 \%$ reduziram o uso de gordura; $51 \%$ reduziram o uso de pão; $58,8 \%$ reduziram o uso de farinha; $11,8 \%$ deixaram de fumar; $13,7 \%$ deixaram de beber e $51 \%$ passaram a realizar atividades físicas.

Os dados referentes ao perfil de uso de medicamentos estão descritos na Tabela 2.

Tabela 2: Características sobre o uso de medicamentos

\begin{tabular}{lcc}
\hline \multicolumn{1}{c}{ Variável } & $\mathbf{n}$ & $\%$ \\
\hline Sobre o uso de medicamentos: & & \\
Somente quando o médico recomenda & 27 & 46,6 \\
Reutiliza uma receita médica antiga & 11 & 19,0 \\
Quando o vizinho/familiar indica ou dá o remédio & 17 & 29,3 \\
Compra diretamente na farmácia por conta própria & 37 & 63,8 \\
Compra diretamente na farmácia por indicação do vendedor & 19 & 32,8 \\
Não usa medicamento, mesmo quando indicado por médico/enfermeiro & 0 & 0
\end{tabular}

Em qual(is) ocasião(ões) se automedica

Dor de cabeça

Febre

Gripe ou resfriado

$28 \quad 53,8$

Enjoo

Náuseas

$07 \quad 13,5$

Diarréia

$12 \quad 23,1$

Outros

Se já utilizou receitas médicas antigas para automedicar-se

Sim

1831,0

Não

4069,0

Se é influenciado por meios de comunicação (jornais, revistas, TV) para a escolha de um medicamento?

Sim

$07 \quad 12,1$ 
Motivos para se automedicar

Já tinha experiência com o medicamento

O medicamento foi utilizado por um conhecido

Todos utilizam este medicamento

Considerou que o que sentia era insignificante

O medicamento estava ao meu alcance imediato

Prefere ir à farmácia diretamente

Falta dinheiro para ir ao médico

Influências da mídia

Por crença religiosa

Destino da medicação não utilizada

Descarte no lixo comum ou no vaso sanitário

Armazena para usar quando precisar

Doação para alguém

Devolução à unidade de saúde

Para a ingestão do medicamento:

Água

5696,6

Suco

Leite

Misturado na comida

Puro

Sobre o horário para tomar o(s) medicamento(s):

Tomo sempre na hora indicada pelo médico/enfermeiro $\quad 39 \quad 67,2$

$\begin{array}{ll}\text { Tomo quando eu lembro } & 08 \quad 13,8\end{array}$

Peço ajuda para alguém me lembrar da hora $\quad 03 \quad 5,2$

Não tomo todos os dias, só quando estou me sentindo mal $12 \quad 20,7$

Fonte: Pesquisa de campo (2014)

Na Tabela 3 é apresentada a distribuição do perfil de automedicação relacionada ao sexo, onde $56,4 \%$ dos funcionários compram diretamente na farmácia por conta própria e entre as funcionárias $78,9 \%$.

Tabela 3: Perfil de automedicação relacionada ao sexo

\begin{tabular}{|c|c|c|c|c|}
\hline \multirow[b]{2}{*}{ Variável (Motivação para o uso de medicamentos) } & \multicolumn{4}{|c|}{ Sexo } \\
\hline & \multicolumn{2}{|c|}{$\begin{array}{c}\text { Feminino (19 no } \\
\text { total) }\end{array}$} & \multicolumn{2}{|c|}{$\begin{array}{c}\text { Masculino (39 no } \\
\text { total) }\end{array}$} \\
\hline Somente quando o médico recomenda & $\begin{array}{c}\mathrm{n} \\
07\end{array}$ & $\begin{array}{c}\% \\
36,8\end{array}$ & $\begin{array}{c}n \\
20\end{array}$ & $\begin{array}{c}\% \\
51,3\end{array}$ \\
\hline Reutiliza uma receita médica antiga & 06 & 31,58 & 05 & 12,8 \\
\hline Quando o vizinho/familiar indica ou dá o remédio & 07 & 36,8 & 10 & 25,6 \\
\hline Compra diretamente na farmácia por conta própria & 15 & 78,9 & 22 & 56,4 \\
\hline $\begin{array}{c}\text { Compra diretamente na farmácia por indicação do } \\
\text { vendedor }\end{array}$ & 8 & 42,1 & 11 & 28,2 \\
\hline
\end{tabular}

O perfil de automedicação relacionada a renda é apresentado na Tabela 4. Uma das motivações para o uso de medicamentos para quem recebe quatro ou mais salários em $72,7 \%$ é somente quando o médico recomenda. Nas demais faixas de renda a principal motivação é comprar diretamente na farmácia por conta própria. 
Tabela 4: Perfil de automedicação relacionada a renda

\begin{tabular}{|c|c|c|c|c|c|c|c|c|}
\hline \multirow{3}{*}{$\begin{array}{l}\text { Variável (Motivação para o } \\
\text { uso de medicamentos) }\end{array}$} & \multicolumn{8}{|c|}{ Renda } \\
\hline & \multicolumn{2}{|c|}{$\begin{array}{c}\text { 1salário } \\
\text { mínimo (10 } \\
\text { no total) }\end{array}$} & \multicolumn{2}{|c|}{$\begin{array}{c}2 \text { salários } \\
\text { mínimos ( } 23 \\
\text { no total) }\end{array}$} & \multicolumn{2}{|c|}{$\begin{array}{c}3 \text { salários } \\
\text { mínimos (14 } \\
\text { no total) }\end{array}$} & \multicolumn{2}{|c|}{$\begin{array}{c}4 \text { ou mais } \\
\text { salários (11 } \\
\text { no total) }\end{array}$} \\
\hline & $\mathrm{n}$ & $\%$ & $\mathrm{n}$ & $\%$ & $\mathrm{n}$ & $\%$ & $\mathrm{n}$ & $\%$ \\
\hline $\begin{array}{l}\text { Somente quando o médico } \\
\text { recomenda }\end{array}$ & 5 & 50 & 7 & 30,4 & 7 & 50 & 8 & 72,7 \\
\hline $\begin{array}{l}\text { Reutiliza uma receita médica } \\
\text { antiga }\end{array}$ & 2 & 20 & 5 & 21,7 & 2 & 14,3 & 2 & 18,18 \\
\hline $\begin{array}{l}\text { Quando o vizinho/familiar } \\
\text { indica ou dá o remédio }\end{array}$ & 4 & 40 & 7 & 30,4 & 4 & 28,5 & 2 & 18,18 \\
\hline $\begin{array}{l}\text { Compra diretamente na } \\
\text { farmácia por conta própria }\end{array}$ & 8 & 80 & 14 & 60,9 & 10 & 71,4 & 5 & 45,45 \\
\hline $\begin{array}{l}\text { Compra diretamente na } \\
\text { farmácia por indicação do } \\
\text { vendedor }\end{array}$ & 4 & 40 & 7 & 30,4 & 5 & 35,7 & 3 & 27,2 \\
\hline
\end{tabular}

Fonte: Pesquisa de campo (2014)

O perfil de automedicação relacionada à cobertura por plano de saúde é descrito na Tabela 5. Quem compra diretamente na farmácia por conta própria representa 66,7\% dos que tem plano de saúde.

Tabela 5: Perfil de automedicação relacionada à cobertura por plano de saúde

\begin{tabular}{l|llll}
\hline \multirow{2}{*}{ Variável (Motivação para o uso de medicamentos) } & \multicolumn{4}{c}{ Plano de Saúde } \\
\cline { 2 - 5 } & \multicolumn{2}{c}{$\begin{array}{c}\text { Sim (30 no } \\
\text { total) }\end{array}$} & Não (28 no total) \\
\hline Somente quando o médico recomenda & $\mathrm{n}$ & $\%$ & $\mathrm{n}$ & $\%$ \\
\hline Reutiliza uma receita médica antiga & 12 & 40 & 15 & 53,57 \\
\hline Quando o vizinho/familiar indica ou dá o remédio & 7 & 23,3 & 4 & 14,3 \\
\hline Compra diretamente na farmácia por conta própria & 13 & 43,3 & 4 & 14,3 \\
\hline Compra diretamente na farmácia por indicação do vendedor & 20 & 66,7 & 17 & 60,7 \\
\hline
\end{tabular}

Fonte: Pesquisa de campo (2014)

O perfil de automedicação relacionada à escolaridade é apresentado na Tabela 6.

Tabela 6: Perfil de automedicação relacionada à escolaridade

\begin{tabular}{lllll}
\hline & \multicolumn{5}{c}{ Escolaridade } \\
\hline \multicolumn{1}{c}{$\begin{array}{l}\text { Variável (Motivação para o uso de } \\
\text { medicamentos) }\end{array}$} & $\begin{array}{c}\mathbf{1} \text { a } \mathbf{8} \text { anos de estudo } \\
(\mathbf{1 0} \text { no total) }\end{array}$ & $\begin{array}{c}\mathbf{9} \text { ou mais anos de estudo } \\
\text { (48 no total) }\end{array}$ \\
\hline & $\mathrm{n}$ & $\%$ & $\mathrm{n}$ & $\%$ \\
\hline Somente quando o médico recomenda & 7 & 70 & 20 & 41,7 \\
\hline Reutiliza uma receita médica antiga & 1 & 10 & 10 & 20,8 \\
\hline $\begin{array}{l}\text { Quando o vizinho/familiar indica ou dá o } \\
\text { remédio }\end{array}$ & 2 & 20 & 15 & 31,25 \\
\hline $\begin{array}{l}\text { Compra diretamente na farmácia por conta } \\
\text { própria }\end{array}$ & 6 & 60 & 31 & 64,6 \\
$\begin{array}{l}\text { Compra diretamente na farmácia por } \\
\text { indicação do vendedor }\end{array}$ & 1 & 10 & 18 & 37,5 \\
\hline
\end{tabular}

Fonte: Pesquisa de campo (2014)

Quem tem de um a oito anos de estudo só tem motivação para o uso de medicamentos somente quando o médico recomenda. Já os funcionários com nove ou mais anos de estudo só tem motivação quem compra diretamente na farmácia por conta própria

\section{Discussão}


O medicamento passou a ser visto como símbolo de saúde (LEFÈVRE, 1987). Ao fazer uso de fármacos, o indivíduo busca um resultado rápido que obscurece outras dimensões da saúde, entendida como "estado de completo bem-estar físico, mental e social e não meramente a ausência de doença ou enfermidade" (DECLARAÇÃO DE ALMA ATA, 1978).

A automedicação estabelece uma prática universal, presente em diferente intensidade nas mais diversas sociedades e culturas, independentemente do grau de desenvolvimento socioeconômico das próprias. Uma revisão sistemática indicou que significante proporção da população adulta brasileira se automedica (DOMINGUES et al, 2015).

Este estudo identificou a prevalência de homens na pesquisa $(67,2 \%)$ com faixa etária de mais de 44 anos $(30,8 \%)$. As mulheres apresentaram prevalência de automedicação maior do que os homens, resultado distinto do que pode ser observado na literatura (DOMINGUES et al, 2015). No tocante à escolaridade, observou-se que $82,8 \%$ dos entrevistados tiveram mais de 9 anos de estudo. A Tabela 1 apresentou os dados referentes a automedicação e escolaridade condizentes com a literatura, quais sejam, que os maiores adeptos da automedicação são aqueles que dispõem de um maior grau de informação. Demonstra-se que o acúmulo de conhecimento, seja ele adquirido nas instituições educacionais ou em experiências de vida, gera uma maior confiança naqueles que se automedicam (VILARINO, 1998).

A média salarial familiar foi de 2 salários mínimos (39,7\%). Damasceno et al. (2007), observou que a renda familiar não influenciou a prática da automedicação, o que corrobora com os achados desta pesquisa, a qual assim como nessa pesquisa que também não apresentou associação significativa quando comparado à classificação socioeconômica com a prática da automedicação. Já no Chile, houve diferença significativa entre automedicação e os vários estratos socioeconômicos, ou seja, $72 \%$ dos sujeitos de classe socioeconômica baixa se automedicavam (SOIBELMAN, 1986).

Os dados obtidos demonstram que $89,7 \%$ dos entrevistados já realizaram a prática de automedicação. Como o estudo não limitou o tempo recordatório, a frequência de automedicação é bastante superior quando comparada com estudos que avaliaram um período de 15 dias antes da aplicação do questionário, que apontam automedicação em um terço da população adulta brasileira (DOMINGUES et al, 2015). Dentre as ocasiões em que o indivíduo se automedica foram relatadas as situações de "dor de cabeça" $(82,7 \%)$, "febre" (61,5\%) e "gripe ou resfriado" $(53,8 \%)$, considerando que a variável para estas respostas foi de múltipla escolha. Entre os motivos que influenciaram na automedicação os mais citados foram a compra diretamente na farmácia por conta própria $(63,8 \%)$ e compra diretamente na farmácia por indicação do vendedor $(32,8 \%)$.Em nosso país, os principais medicamentos para as citadas afecções podem ser comprados sem receita médica, facilitando o acesso e consequentemente a prática de automedicação.

Experiências exitosas com determinados medicamentos também podem favorecer a prática de automedicação. Este estudo demonstrou que $65,5 \%$ dos entrevistados utilizam sobras de medicamentos oriundas de tratamentos anteriores. Se considerarmos a variável de múltipla escolha "Motivos para se automedicar", $88,7 \%$ proferiram que já tinham experiência com o medicamento. Resultados semelhantes foram encontrados na cidade de Porto Alegre (RS), onde $57,14 \%$ das pessoas escolheram seus medicamentos baseados em experiências passadas (VITOR et al., 2008). O perfil epidemiológico da automedicação no Brasil mostrou que $51 \%$ das pessoas se automedicavam baseados em sugestões de pessoas não qualificadas e 40,0\% em prescrições anteriores (ARRAIS, 1997). Já em Portugal, $50,0 \%$ dos medicamentos utilizados na automedicação foram aconselhados pelo farmacêutico e 1,4\% por indicação do enfermeiro (MENDES et al., 2004). Estes resultados são importantes, pois, podem orientar as estratégicas para a 
promoção do uso racional de medicamentos dependendo do público que se pretende atuar.

Mesmo que a automedicação faça parte do autocuidado é importante que seja avaliado se a mesma é realizada de maneira responsável. Apesar de não ser um acontecimento único do mundo moderno, o uso de medicamentos sem prescrição médica torna-se uma prática banal na população brasileira em todos os grupos etários (ARRAIS, 1997). Interessante destacar que $86,8 \%$ dos entrevistados afirmaram insatisfação com relação ao atendimento de saúde pública. O trabalho de Schmid et al. (2010), realizado em São Paulo, mostra que a probabilidade da prática de automedicação por uma pessoa com morbidade aguda que tem acesso ao atendimento público de saúde era de $15 \%$, enquanto que dentre os que não têm esse atendimento a probabilidade era de $68 \%$, ficando clara a importância do acesso aos serviços de saúde. Outro dado observado foi que pessoas com planos de saúde automedicam-se de forma mais significativa: 66,7\% dos entrevistados que tem plano de saúde afirmam comprar medicamentos na farmácia por indicação de vendedor. Este resultado é controverso daqueles apresentados por Vilarino (1998) em seu estudo com a população em geral. Uma das hipóteses levantadas para explicar este fenômeno seria o fato de que pessoas que possuem planos de saúde provavelmente reutilizam prescrições mais antigas, hipótese que não foi confirmada neste trabalho, pois $69 \%$ dos entrevistados afirmaram nunca terem utilizado receitas médicas antigas para se automedicar.

A maioria dos entrevistados (74\% do público da pesquisa) demonstra não possuir bom nível de informação para a utilização de medicamentos. Muitos deles utilizam o medicamento sem ao menos saber para que servem, pois afirmam não lerem a bula dos medicamentos adquiridos através de automedicação. Quando os medicamentos são receitados pelo médico, $67,2 \%$ utilizam o medicamento de acordo com a prescrição, índice que também pode ser considerado aquém do ideal.

Outro achado controverso da pesquisa foi a demonstração que $87,9 \%$ dos entrevistados não se declaram influenciados por propagandas. Nascimento (2007) demonstrou que o alto índice de automedicação da população brasileira tem forte relação com o mercado ocupado pela indústria farmacêutica, que não mede esforços para estimular o consumo de medicamentos através das ferramentas da mídia (NASCIMENTO, 2007).

Outro dado importante da pesquisa foi o relato de $77,6 \%$ dos entrevistados, que afirmam recorrerem ao uso de folhas, chás e plantas medicinais por acreditarem que estes não promovem danos à saúde. Mesmo com o estímulo pela OMS no uso da medicina complementar/alternativa nos sistemas de saúde de forma integrada com as técnicas da medicina ocidental (MICHILES, 2004), fica evidente a necessidade de orientação quanto ao uso deste tipo de fármaco, haja vista o relato de aumento do número de reações adversas possivelmente em razão do cada vez maior interesse populacional pelas terapias naturais (SILVEIRA et al, 2008).

O presente estudo contribuiu para melhor conhecimento do fenômeno da automedicação dentre os funcionários terceirizados do CCS/UFRB, bem como para reflexão sobre trabalhar em um espaço de ensino/aprendizagem em saúde e a possibilidade de aquisição de conhecimentos sobre o próprio bem-estar e saúde.

\section{Conclusão}

Segundo a OMS (WORLD HEALTH ORGANIZATION, 1987), propõe-se que para o uso racional de medicamentos é preciso, primeiramente, estabelecer a necessidade do uso do medicamento, em seguida, que se receite o medicamento apropriado, a melhor escolha, de acordo com os conselhos de eficácia e segurança comprovados e aceitáveis. Do mesmo modo, é necessário que o medicamento seja prescrito adequadamente, na 
forma farmacêutica, doses e período de duração do tratamento, que esteja disponível, a um preço acessível, e que responda sempre aos critérios de qualidade exigidos, que se dispense em condições adequadas, com a necessária orientação e responsabilidade, e, finalmente, que se cumpra o regime terapêutico já prescrito, da melhor maneira possível.

Os resultados obtidos corroboram com a literatura: o índice de automedicação no Brasil é alarmante, quer seja pela falta de conhecimento sobre os medicamentos (e o risco do uso dos mesmos), quer seja pelo fácil acesso aos mesmos. O estudo também demonstrou que a dificuldade de acesso ao serviço de saúde pública favorece a busca pela terapêutica medicamentosa, que ocorre tanto pelo resgate de experiências ditas exitosas, quanto pela indicação de medicamentos por amigos, familiares ou até mesmo por balconistas.

Os resultados demonstram que a maioria dos funcionários terceirizados do CCS/UFRB faz uso de medicação por conta própria, incluindo-se o uso de fitoterápicos. 0 presente estudo usou de uma pesquisa que não investigava diretamente a automedicação, e sim o perfil da utilização de medicamentos, o que pode levar a resultados distintos dos que seriam obtidos em uma pesquisa específica sobre o tema. Contudo, a conexão com resultados anteriores, tanto no que tange as variáveis sócio demográficas, quanto às características sobre o uso de medicamentos utilizados, implica que os achados refletem a realidade vivenciada pela população estudada. Desta forma, conclui-se que os trabalhadores terceirizados de um centro universitário formador de profissionais de saúde não têm acesso a informações essenciais sobre autocuidado e saúde, o que nos leva a sugerir a execução de projetos que visem a educação continuada em saúde para estes trabalhadores.

\section{Referências}

ARRAIS, P. S. D., Perfil da automedicação no Brasil. Revista de Saúde Pública, v. 31, n. 1, p. 71-7, 1997.

BARROS, J. A. C. A atuação dos balconistas de farmácia: ajudando a promover saúde? Journal Brasileiro de Medicina, v. 73, n. 2, p. 120-7, 1997.

BOCK, L.; TARANTINO, M. Atração: o brasileiro exagera nos remédios, consumindo-os sem consultar o médico e colocando sua saúde em risco. Isto é., v. 1671, p. 80-85, 2001.

CAMPOS, J. A.; et al. Prescrição de medicamentos por balconistas de 72 farmácias de Belo horizonte/ MG em maio de 1983: Riscos e Acidentes. Journal de Pediatria, v. 59, n. 3, p. 307-312. ago. 1985.

CIPOLLE, D. J.; STRAND, L. M.; MORLEY, P. C. El ejercicio de la atención farmacéutica. Madrid: McGraw Hill, Interamericana, p. 1-36, 2000.

DAMASCENO, D. D.; et al. Automedicação entre graduandos de Enfermagem, Farmácia e Odontologia da Universidade Federal de Alfenas. Revista Mineira de Enfermagem, v. 11, n. 1, p. 48-52, jan/mar, 2007.

DECLARACÃ̃O DE ALMA-ATA. Conferência Internacional sobre cuidados primários de saúde; 6-12 de setembro 1978; Alma-Ata; USSR. In: Ministério da Saúde (BR). Secretaria de Políticas de Saúde. Projeto Promoção da Saúde. Declaração de Alma-Ata; Carta de Ottawa; Declaração de Adelaide; Declaração de Sundsvall; Declaração de 
Santafé de Bogotá; Declaração de Jacarta; Rede de Megapaíses; Declaração do México. Brasília (DF): Ministério da Saúde; 2001. p. 15.

DOMINGUES, P. H. F.; et al. Prevalência da automedicação na população adulta do Brasil: revisão sistemática. Revista de Saúde Pública, vol.49, n. 36, p. 1-8, 2015.

FAUS, M. J. Atención farmacéutica como respuesta a uma necesidad social. Ars Pharmaceutica, v.41, n. 1, p. 137-143, 2000.

FUNDAÇÃO OSWALDO CRUZ/CENTRO DE INFORMAÇÃO CIENTÍFICA E TECNOLÓGICA/SISTEMA NACIONAL DE INFORMAÇÕES TÓXICOFARMACOLÓGICAS. Estatística Anual de Casos de Intoxicação e Envenenamento. Brasil, 2016. Disponível em: <https://sinitox.icict.fiocruz.br/dados-nacionais>. Acesso em: 28 dez. 2018.

JOHNSON, J. A., BOOTMAN, J. L. Drug related morbidity and mortality. A cost -of illness model. Arch Intern Med, v. 155, p. 1949-1956, oct. 1995.

KELLY, J. M. Implementing a Patient Self- Medication Program. Rehabilitation Nursing, v.19, p. 87-90, 1994.

LEFÈVRE, F. A oferta e a procura de saúde através do medicamento: proposta de um campo de pesquisa. Revista de Saúde Pública, v.21, p. 64-7, 1987.

LIMAYE, D. A Systematic Review of the Literature to Assess Self-medication Practices. Ann Med Health Sci Res. 7: 1-15, 2017.

MALHOTRA, S., JAIN, S., PANDHI, P., Drug - related visits to the medical emergency department: a prospective study from India. International Journal of Clinical Pharmcology Therapeutics, v. 39, p. 12-18, jan. 2001.

MARIN, N. Assistência farmacêutica para gerentes municipais. Organizado por Nelly Marin et al. Rio de Janeiro: OPAS/OMS, 2003.

MENDES, Z.; et al. Prevalência da automedicação na população urbana portuguesa. Revista Brasileira de Ciências Farmacêuticas, v. 40, n. 1, 2004.

MICHILES, E. Diagnóstico situacional dos serviços de fitoterapia no estado do Rio de Janeiro. Revista Brasileira de Farmacognosia, v. 14, p. 16-19, 2004.

MILIÁN, A. J. G.; MARTÍNEZ, I. D.; BASTANZURI, C. L. Estrategia para lograr un uso racional de los medicamentos. Revista Cubana de Medicina General Integrada, Cuba, v. 19, n. 2, mar./abr. 2003.

NASCIMENTO, A. Propaganda de medicamentos: como conciliar uso racional e a permanente necessidade de expandir mercado? Trabalho Educação e Saúde, v. 5, n. 2, p. 189-250, 2007.

NASCIMENTO, M.C. Medicamentos: ameaça ou apoio à saúde? Rio de Janeiro: ed. Vieira e Lent; 2003. 
NAVES, J. O. S.; et al. Automedicação: uma abordagem qualitativa de suas motivações. Ciência e Saúde Coletiva, v. 15, n. 1, p.1751-1762, 2010.

PAULO, L.G.; ZANINE, A.C. Automedicação no Brasil. Revista Associação Médica Brasileira, v . 34, p. 69-75, 1988.

PERINI, E.; MODENA, C.M.; RODRIGUES, R.N. et al. Consumo de Medicamentos e adesão às prescrições: objeto e problema de epidemiologia. Revista Ciências Farmacêuticas, v. 20, p. 471-488, 1999.

SCHMID, B; BERNAL, R.; SILVA, N. N. Automedicação em adultos de baixa renda no município de São Paulo. Revista Saúde Pública, v. 44, n. 6, p.1039-45, 2010.

SILVEIRA, P.F.; BANDEIRA, M.A.M.; ARRAIS, P.S.D. Farmacovigilância e reações adversas às plantas medicinais e fitoterápicos: uma realidade. Revista Brasileira de Farmacognosia. 18(4): 618-626, Out./Dez. 2008

SOIBELMAN, M.; AMARAL, L.R.; PALMINI, A.F. Indicações de medicamentos por balconistas de farmácias em Porto Alegre, RS. Revista Associação Médica Brasileira, v. 32, n. 5/6, p. 79-83, 1986.

VILARINO, J. F. Perfil da automedicação em município do Sul do Brasil. Revista Saúde Pública, v. 32, n. 1, p. 43-9, 1998.

VITOR, R. S.; LOPES, C. P.; MENEZES, H. S., KERKHOFF, C.S. E. Padrão de consumo de medicamentos sem prescrição médica na cidade de Porto Alegre, RS. Ciência e Saúde Coletiva, v.13, p. 737-743, 2008.

WORLD HEALTH ORGANIZATION. The rational use of drugs: report of the conference of experts. Nairobi 1985 Jul p. 25-29. Geneva: WHO; 1987. 